\title{
$\infty \sqrt{1}$ Stroke and \\ Role of the glymphatic system in ageing and diabetes mellitus impaired cognitive function
}

\author{
Li Zhang, ${ }^{\oplus}$ Michael Chopp, ${ }^{1,2}$ Quan Jiang, ${ }^{\oplus 1}$ Zhenggang Zhang ${ }^{1}$
}

To cite: Zhang L, Chopp M, Jiang $Q$, et al. Role of the glymphatic system in ageing and diabetes mellitus impaired cognitive function. Stroke and Vascular Neurology 2019;4: e000203. doi:10.1136/svn2018-000203

Received 26 0ctober 2018 Revised 4 January 2019 Accepted 17 April 2019 Published Online First 10 May 2019
Check for updates

(C) Author(s) (or their employer(s)) 2019. Re-use permitted under CC BY-NC. No commercial re-use. See rights and permissions. Published by BMJ.

${ }^{1}$ Department of Neurology, Henry Ford Hospital, Detroit, Michigan, USA

${ }^{2}$ Department of Physics, Oakland University, Rochester, Michigan, USA

Correspondence to Dr Li Zhang; Izhang3@hfhs.org

\section{ABSTRACT}

Diabetes mellitus (DM) is a common metabolic disease in the middle-aged and older population, and is associated with cognitive impairment and an increased risk of developing dementia. The glymphatic system is a recently characterised brain-wide cerebrospinal fluid and interstitial fluid drainage pathway that enables the clearance of interstitial metabolic waste from the brain parenchyma. Emerging data suggest that DM and ageing impair the glymphatic system, leading to accumulation of metabolic wastes including amyloid- $\beta$ within the brain parenchyma, and consequently provoking cognitive dysfunction. In this review, we concisely discuss recent findings regarding the role of the glymphatic system in DM and ageing associated cognitive impairment.

\section{GLYMPHATIC SYSTEM}

The mammalian brain has the highest energy demand of the whole body. With merely $2 \%$ of the body weight, human brain consumes $\sim 20 \%$ of an individual's oxygen and glucose supply to maintain physiological brain function. Given high metabolic activity, effective elimination of the metabolic wastes is essential for homoeostasis of the microenvironment of brain cells. In the peripheral tissue, the lymphatic system collects the interstitial fluids that contain the metabolites, and enables the clearance of excessive metabolic wastes. However, as the central nervous system (CNS) lacks of classic lymphatic vessels, the clearance of metabolic waste products from brain has long been presumed solely via the cerebrospinal fluid (CSF) drainage pathways from the subarachnoid space mainly through arachnoid villi into the venous sinuses, and via cranial nerve sheaths into the peripheral lymphatic system. ${ }^{1}$ The anatomical structures and mechanisms that regulate the clearance of parenchyma interstitial fluid (ISF) and solutes remain less clear. ${ }^{2}$ With advanced in vivo imaging techniques, emerging data have revealed the presence of a clearance system, referred to as the glymphatic system, which clears extracellular solutes from the brain. ${ }^{4-7}$

The glymphatic system is a brain-wide CSF/ ISF drainage system that enables the clearance of interstitial metabolic waste from the brain parenchyma. In vivo imaging studies in rodents show that arterial pulsations drive CSF into the brain parenchyma from the subarachnoid space via the abluminal side of penetrating arteries/ arterioles. ${ }^{458}$ Once CSF arrives at the microvascular level, aquaporin-4 (AQP4) channels located on the ensheathed astrocytic end-feet facilitate the convective flow movement of CSF containing water, ions and small molecule solutes including metabolic wastes into the interstitial space. These wastes then exit from the parenchyma via the paravenous spaces. ${ }^{910}$ Ultimately, the excessive fluids and metabolic wastes are collected, at least partly, into deep cervical lymphatics that are adjacent to the internal jugular veins. ${ }^{4}$ Recent experiments show that at least a portion of drained interstitial fluids and solutes re-enter subarachnoid spaces, where the metabolic wastes including amyloid- $\beta$ were collected by meningeal lymphatic vessels, or enter the classical subarachnoid CSF drainage pathways via arachnoid villi and/or para-nerve sheathes into peripheral lymphatics. ${ }^{11}$ Given its role on brain waste clearance that resembles peripheral lymphatic system and the importance of astrocytic AQP4 facilitated parenchyma CSF/ ISF transport, this brain-wide CSF/ISF drainage pathway was named glymphatic (glia-lymphatic) system $^{4}$ (please see figure 1 for a schematic illustration of the glymphatic system). The waste clearance functions of glymphatic system have been further characterised. AQP4 gene deletion leads to profound reductions of interstitial influx of paravascular solutes and the clearance of interstitial solutes such as amyloid- $\beta$, indicating the pivotal role of astrocytic AQP4 in glymphatic transport. ${ }^{4}$ During sleep, the interstitial space volume in mouse expands $60 \%$ over that in the awake state, which lowers resistance to the influx of para-arterial CSF to interstitial space, and therefore, accelerate fluxes of CSF/ ISF along the glymphatic pathway and robustly enhances interstitial neurotoxic metabolite clearance including amyloid- $\beta .^{5}$ Additionally, different body postures and physiological pulsations evoked by respiratory and cardiac 


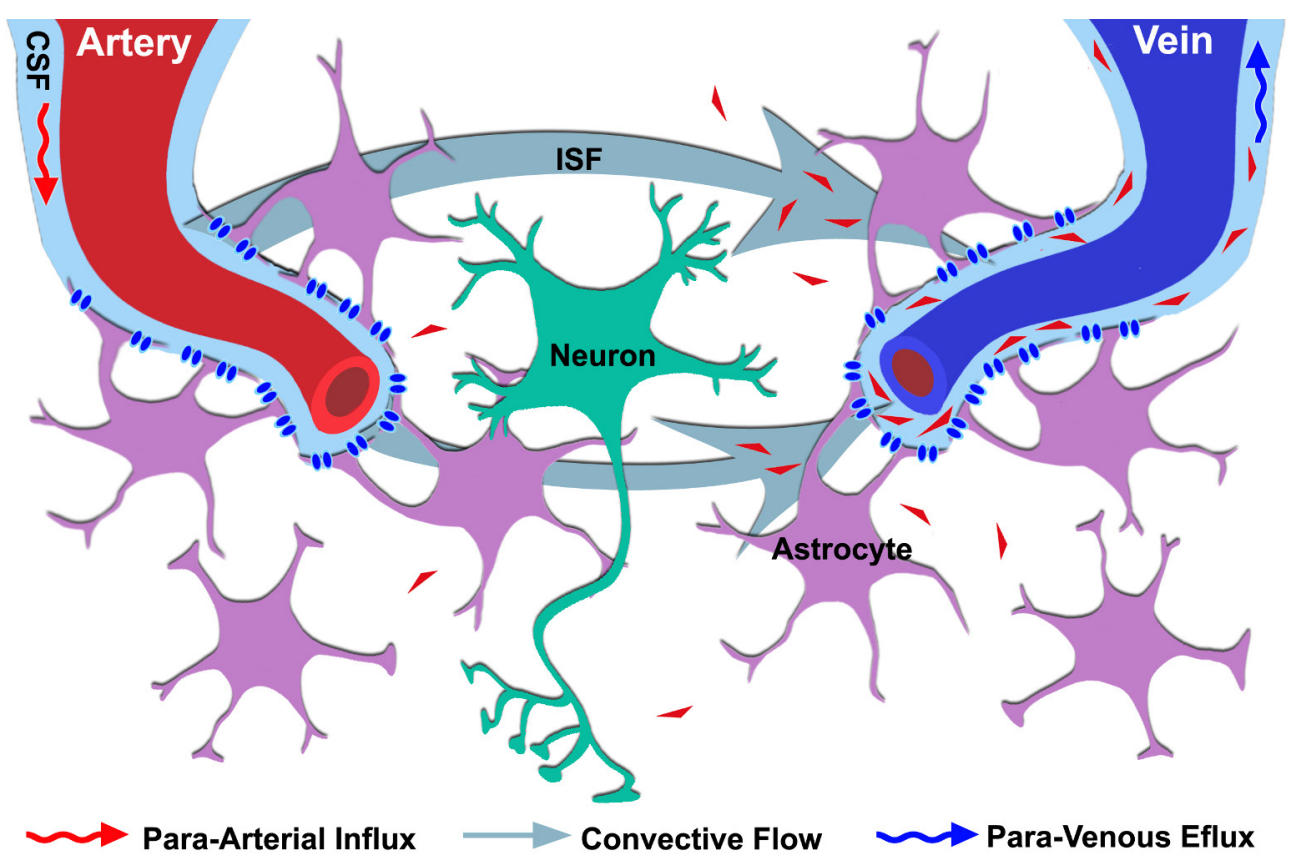

0 io Aquaporin-4 Channels

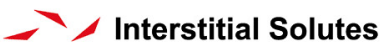

Figure 1 Overview of glymphatic system. CSF enter the brain parenchyma along the para-arterial space. The AQP4 channels located on the astrocytic end-feet facilitate the convective flow movement of CSF-ISF within the interstitial space. The excessive ISF and its containing solutes then exit from the parenchyma via the para-venous spaces. AQP4, aquaporin-4; CSF, cerebrospinal fluid; ISF, interstitial fluid.

rhythm also influence the glymphatic drainage function. ${ }^{12} 13$ Collectively, accumulating experimental data support the important role of the glymphatic system hypothesis in regulating brain metabolites clearance.

\section{GLYMPHATIC SYSTEM IN AGEING AND DM ASSOCIATED COGNITIVE DECLINE}

$\mathrm{DM}$ is a common metabolic disease in the middle-aged and older population, which is associated with cognitive decline and an increased risk of developing dementia in the elderly. ${ }^{14-17}$ While advanced age remains a prominent risk factor for cognitive decline and dementia, DM accelerates the cognitive impairment and increases the risk for dementia in the elderly. ${ }^{14}$ Given the multifaceted nature of the metabolic disease, multiple pathological mechanisms have been implicated in the development of cognitive abnormalities, such as cerebral vascular complications, disturbances of glucose homoeostasis, oxidative stress and neurotoxicity, and defective in amyloid metabolism. ${ }^{18}$ Emerging evidence also suggests that DM and ageing are associated with a dramatic decline in glymphatic function, which may negatively affect cognitive function.

In the normal aged brain, several structural and functional abnormalities in the glymphatic system including the reduction of cerebrovascular pulsatility and the depolarisation of astrocytic AQP4 hamper glymphatic system function. ${ }^{8}$ As parenchymal accumulation of metabolic wastes including amyloid- $\beta$ is an established pathological substrate in cognitive decline during ageing and Alzheimer's disease, ${ }^{19} 20$ it was postulated that glymphatic dysfunction contributes to ageing associated cognitive decline via impairing the parenchyma amyloid- $\beta$ clearance. ${ }^{8}$ Indeed, in an animal model of Alzheimer's disease, the reduction of glymphatic transport preceded to the appearance of parenchymal amyloid- $\beta$ deposits, suggesting that glymphatic dysfunction may be a causative factor for Alzheimer's disease pathology. ${ }^{21}$

The prevalence of cognitive dysfunction is high in patients with diabetes, especially among the elderly. ${ }^{22}$ However, due to lack of animal models, there are few preclinical studies to investigate the effect of DM on glymphatic system function and its contributions to cognitive impairment during ageing. Using a chemically induced DM model in the middle-aged rats, a recent study showed that DM accelerates the progression of hippocampal related cognitive decline that mimics the clinical deterioration of cognitive dysfunction observed in aged patients with type 2DM. ${ }^{23}$ Subsequent study showed that after intracistern magna injection of small molecule contrast agent gadolinium-diethylene-triaminepentaacetic acid (Gd-DTPA) and CSF/ISF fluorescence tracer, MRI and ex vivo fluorescence microscopic imaging studies reveal that glymphatic drainage of MRI and fluorescence tracers in the hippocampus and hypothalamus are profoundly reduced in the middle-aged DM rats, which are highly correlated with the learning and memory deficits. ${ }^{23}$ In parallel, DM induced cognitive function impairment in the middle-aged rats was associated with hippocampal astrocyte activation, loss of paravascular AQP4 immunoreactivity and microvascular disruption, all of which are 
key anatomical and functional components which facilitate glymphatic system function. ${ }^{24}$ Together, the observed correlation between the hippocampal glymphatic dysfunction and the cognitive deficits in the middle-aged DM rats emphasises the key role of glymphatic system in DM and ageing associated cognitive deficits. However, as ageing and DM associated cognitive dysfunctions are attributed to a dynamic accumulation of various neuropathogenic processes, the dysfunction of glymphatic system may represent a composite of neuropathological alterations that underlie ageing and DM associated cognitive deficits.

The physiological and pathophysiological significance of the glymphatic system in the brain is being actively investigated. There are controversies regarding the anatomical elements and flow dynamics arising from the discovery of glymphatic system. ${ }^{25}{ }^{26}$ Recent evidence on the existence of meningeal lymphatic vessels added another layer of regulatory mechanisms of glymphatic system on cognitive function. ${ }^{311}$ Indeed, a recent study shows while learning and memory impairments observed in aged mouse is accompanied with decreased meningeal lymphatic vessels coverage and impairment of the glymphatic CSF/ISF transport, enhancing meningeal lymphatic drainage with vascular endothelial growth factor $\mathrm{C}$ treatment accelerates parenchymal glymphatic CSF/ISF transport and improves learning and memory performance in the aged mouse. ${ }^{11}$ These data reflect our growing understanding of the complexity of the role glymphatic system and its interaction with other brain drainage mechanisms in regulating cognitive function.

In conclusion, emerging data demonstrate that the glymphatic system mediated CSF-ISF exchange is instrumental in maintaining homoeostasis of the neural microenvironment. Ageing and DM incited impairments of the glymphatic system may predispose to the development of cognitive dysfunction and neurodegenerative diseases. However, in recognition of the complexity of the potential pathological mechanisms implicated in ageing and DM associated cognitive decline, the relative contribution and causative interplay between glymphatic system and other neuropathological factors in DM and ageing induced cognitive deficits warrant further investigation. Defining DM and ageing associated glymphatic system disruption may potentially have important clinical implications in predicting, preventing and providing potential targets for new treatment paradigms for cognitive decline.

Contributors $\mathrm{LZ}$ and ZZ proposed overall structure and drafted manuscript. MC and QJ provided critical feedback and helped shape the manuscript. All authors contributed to the final version of the manuscript.

Funding The authors have not declared a specific grant for this research from any funding agency in the public, commercial or not-for-profit sectors.

Competing interests None declared.

Patient consent for publication Not required.

Provenance and peer review Commissioned; externally peer reviewed.

Open access This is an open access article distributed in accordance with the Creative Commons Attribution Non Commercial (CC BY-NC 4.0) license, which permits others to distribute, remix, adapt, build upon this work non-commercially, and license their derivative works on different terms, provided the original work is properly cited, appropriate credit is given, any changes made indicated, and the use is non-commercial. See: http://creativecommons.org/licenses/by-nc/4.0/.

\section{REFERENCES}

1. Weller RO, Subash M, Preston SD, et al. Perivascular drainage of amyloid-beta peptides from the brain and its failure in cerebral amyloid angiopathy and Alzheimer's disease. Brain Pathol 2008;18:253-66.

2. Aspelund A, Antila S, Proulx ST, et al. A dural lymphatic vascular system that drains brain interstitial fluid and macromolecules. $J$ Exp Med 2015;212:991-9.

3. Louveau A, Smirnov I, Keyes TJ, et al. Structural and functional features of central nervous system lymphatic vessels. Nature 2015;523:337-41.

4. Iliff JJ, Wang M, Liao Y, et al. A paravascular pathway facilitates CSF flow through the brain parenchyma and the clearance of interstitial solutes, including amyloid $\beta$. Sci Trans/ Med 2012;4:147ra111.

5. Xie L, Kang H, Xu Q, et al. Sleep drives metabolite clearance from the adult brain. Science 2013;342:373-7.

6. Ringstad G, Valnes LM, Dale AM, et al. Brain-wide glymphatic enhancement and clearance in humans assessed with MRI. JCl Insight 2018;3.

7. Ringstad G, Vatnehol SAS, Eide PK. Glymphatic MRI in idiopathic normal pressure hydrocephalus. Brain 2017;140:2691-705.

8. Kress BT, Iliff JJ, Xia M, et al. Impairment of paravascular clearance pathways in the aging brain. Ann Neurol 2014;76:845-61.

9. Tarasoff-Conway JM, Carare RO, Osorio RS, et al. Clearance systems in the brain-implications for Alzheimer disease. Nat Rev Neurol 2015;11:457-70.

10. Iliff $\mathrm{JJ}$, Nedergaard M. Is there a cerebral lymphatic system? Stroke 2013;44:S93-S95.

11. Da Mesquita S, Louveau A, Vaccari A, et al. Functional aspects of meningeal lymphatics in ageing and Alzheimer's disease. Nature 2018;560:185-91.

12. Lee $H$, Xie L, Yu M, et al. The effect of body posture on brain glymphatic transport. J Neurosci 2015;35:11034-44.

13. Kiviniemi V, Wang $X$, Korhonen V, et al. Ultra-fast magnetic resonance encephalography of physiological brain activity Glymphatic pulsation mechanisms? J Cereb Blood Flow Metab 2016;36:1033-45.

14. Biessels GJ, Staekenborg S, Brunner E, et al. Risk of dementia in diabetes mellitus: a systematic review. Lancet Neurol 2006;5:64-74.

15. Awad N, Gagnon M, Messier C. The relationship between impaired glucose tolerance, type 2 diabetes, and cognitive function. J Clin Exp Neuropsychol 2004;26:1044-80.

16. Whitmer RA, Sidney S, Selby J, et al. Midlife cardiovascular risk factors and risk of dementia in late life. Neurology 2005;64:277-81.

17. Schnaider Beeri M, Goldbourt U, Silverman JM, et al. Diabetes mellitus in midlife and the risk of dementia three decades later. Neurology 2004;63:1902-7.

18. Qiu C, Sigurdsson S, Zhang Q, et al. Diabetes, markers of brain pathology and cognitive function: the Age, Gene/Environment Susceptibility-Reykjavik Study. Ann Neurol 2014;75:138-46.

19. Iliff JJ, Wang M, Liao Y, et al. A paravascular pathway facilitates csf flow through the brain parenchyma and the clearance of interstitial solutes, including amyloid. Sci Transl Med 2012;4:147ra111.

20. Hardy J, Selkoe DJ. The amyloid hypothesis of alzheimer's disease: Progress and problems on the road to therapeutics. Science (New York, N. Y.) 2002;297:353-6.

21. Peng W, Achariyar TM, Li B, et al. Suppression of glymphatic fluid transport in a mouse model of Alzheimer's disease. Neurobiol Dis 2016;93:215-25.

22. Yaffe K, Blackwell T, Kanaya AM, et al. Diabetes, impaired fasting glucose, and development of cognitive impairment in older women. Neurology 2004;63:658-63.

23. Zhang L, Chopp M, Zhang Y, et al. Diabetes mellitus impairs cognitive function in middle-aged rats and neurological recovery in middle-aged rats after stroke. Stroke 2016;47:2112-8.

24. Jiang $Q$, Zhang L, Ding G, et al. Impairment of the glymphatic system after diabetes. J Cereb Blood Flow Metab 2017;37:1326-37.

25. Morris AW, Sharp MM, Albargothy NJ, et al. Vascular basement membranes as pathways for the passage of fluid into and out of the brain. Acta Neuropathol 2016;131:725-36.

26. Hawkes CA, Jayakody N, Johnston DA, et al. Failure of perivascular drainage of $\beta$-amyloid in cerebral amyloid angiopathy. Brain Pathol 2014;24:396-403. 